\section{Subwavelength Focusing of GHz Radiation by 2D Metamaterials Demonstrated}

Metamaterials, or "left-handed" materials, are artificial structures that exhibit negative permittivity $(\varepsilon)$ and permeability $(\mu)$ for certain frequencies of electromagnetic waves. This property can be utilized for focusing electromagnetic radiation through negative refraction. In particular, a material with $\varepsilon=-1$ and $\mu=$ -1 has been predicted to focus light beyond the diffraction limit. In the March 15 issue of Optics Letters (p. 814), I. Bulu and coworkers at Bilkent University in Ankara, Turkey, have reported subwavelength focusing of $\mathrm{GHz}$ radiation by metamaterials based on a labyrinth structure.

The labyrinth structure was obtained by modifying the splitring resonator structure commonly used in metamaterial systems. It has four concentric rings, $0.15 \mathrm{~mm}$ thick, with two slits on each ring. The unit cell consists of two thin substrates of dimensions $8.8 \mathrm{~mm} \times 8.8 \mathrm{~mm}$ oriented at $90^{\circ}$ with respect to each other. Standard printed circuit board manufacturing methods are used to print the copper labyrinth structure on one side of the substrate and copper wire stripes, $2.5 \mathrm{~mm}$ wide, on the other side. The copper thickness was $0.05 \mathrm{~mm}$. The whole sample has length, width, and height of 68 , five- and 20 -unit cells, respectively. Calculations show that this metamaterial system exhibits a negative refractive index for frequencies ranging from $6.1 \mathrm{GHz}$ to $6.4 \mathrm{GHz}$, with $n=-1$ at around $6.3 \mathrm{GHz}$.

Using monopole antennas as source and receiver, the researchers measured the electric-field intensity over an area of $100 \mathrm{~mm} \times 200 \mathrm{~mm}$ on the output face of the metamaterial. Examination of intensity profiles at $6.3 \mathrm{GHz}$ along the horizontal and vertical directions of the output face revealed that radiation from the source can be focused to a spot size as small as $\lambda / 4$. The researchers said that this demonstration of subwavelength focusing of electromagnetic waves by metamaterials in free space may find useful applications in the field of imaging.

TUSHAR PRASAD

\section{Slow Light Efficiently Coupled Along Bends in Photonic Crystal Waveguide}

Photonic crystals are periodic dielectric structures capable of manipulating light propagation in a variety of novel ways. Unlike conventional optical fibers, waveguides based on photonic crystals can channel light efficiently around very sharp bends and are considered to be the routing devices for the next generation of integrated optics. Straight photonic crystal waveguides allow all waveguide modes to transmit. However, light at wavelengths close to the onset of the waveguiding mode-that is, in the regime of a very large group velocity dispersion that could be consistent with a greatly reduced group velocity (slow light) does not transmit through photonic crystal waveguides with bends. In the March 15 issue of Optics Letters (p. 745), S. Assefa and co-workers at IBM T.J. Watson Research Center in Yorktown Heights, N.Y., have shown that light transmission at wavelengths commensurate with slow light can be improved by optimized design of the photonic crystal waveguide bend.

Using conventional semiconductor processing technology, twodimensional photonic crystals (lattice constant $a=437 \mathrm{~nm}$ ) with a triangular lattice of holes (radius $r=0.23 a$ ) were fabricated on silicon-on-insulator wafers. Waveguides were created by removing a row of holes along the $\Gamma-K$ direction, and up to 20 bends were incorporated into the whole waveguide structure by cascading straight waveguides at $60^{\circ}$ with respect to each other. Transmission measurements estimate the loss per bend to be very low but also reveal that the long-wavelength cutoff is blueshifted by about $30 \mathrm{~nm}$ with respect to a straight waveguide. That blueshift

\section{Precision Heating to $1200^{\circ} \mathrm{C}$ in 24 seconds, for under $\$ 10,000$.}
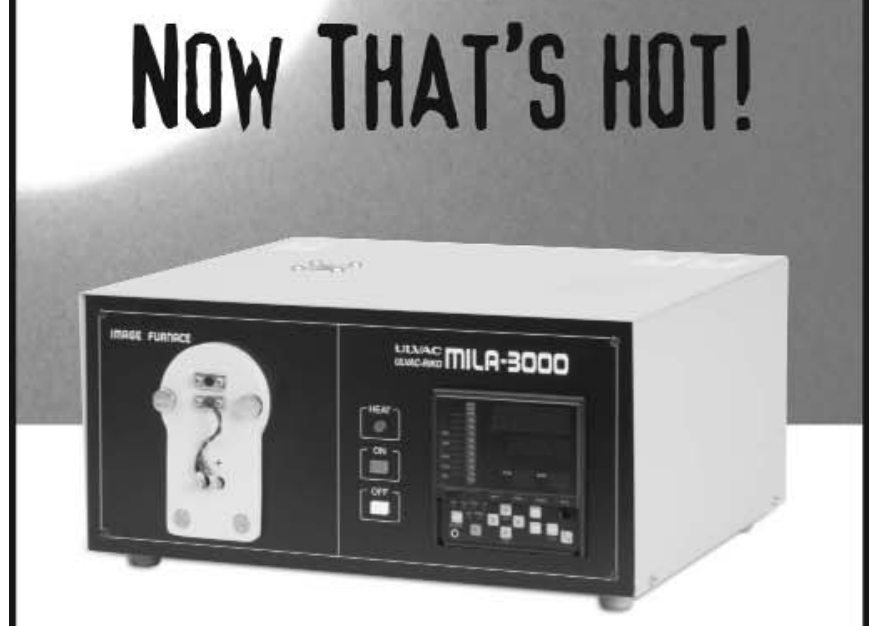

Anneal your small samples faster and with better control using the programmable MILA-3000 tabletop furnace. Ulvac's Mini-Lamp Annealing System can rapidly heat and cool samples with it's infrared gold image furnace, providing precision high temperature control, clean heating and versatile atmosphere selection.

\section{MILA-3000 Features:}

- High controlled heating rates of $50^{\circ} \mathrm{C} / \mathrm{s}$

- Sample size $20 \times 20 \times 20 \mathrm{~mm}$

- Air, vacuum and inert/reactive gas atmosphere

- Temperature uniformity of $+/-2^{\circ} \mathrm{C}$

- Low power consumption (1 kW)

For all your annealing and thermal processes, turn on the heat with the MILA-3000!

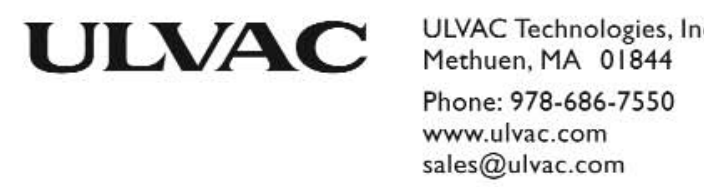


left the regime of possible slow light propagation in an opaque spectral region above the long-wavelength cutoff. To efficiently couple the waveguide modes with longer wavelengths (in the slow light regime) into the bends, the average refractive index at the bend was effectively increased by producing a waveguide with smaller hole radii in the bend vicinity. For waveguide structures with $r=$ $0.33 a$, the blueshift in long-wavelength cutoff was reduced from $15 \mathrm{~nm}$ to $6 \mathrm{~nm}$ when the radii of four holes in the vicinity of the bends were decreased, allowing for some light transmission in the slow light spectral region. The researchers said that the blueshift can be completely compensated and all of the wavelengths commensurate with slow light propagation can be incorporated into the high-transmission region of the waveguide if the refractive index is further increased by decreasing the radii of 16 nearby holes instead of four in the bend vicinity. According to the researchers, this demonstration makes photonic crystal waveguides very promising devices for slow light applications such as all-optical data storage and quantum computing.

TUSHAR PRASAD

\section{Virtual-Detector-Based Photoacoustic Microscopy Improves In Vivo Imaging}

Photoacoustic microscopy (PAM) is a promising, noninvasive imaging technique that shows potential for applications in dermatology and related cancer research. The technique employs the absorption of laser pulses to produce a thermoelastic expansion of biological media and ultimately emitting a photoacoustic (PA) signal. Large-numericalaperture (NA) ultrasound transducers detect the acoustic signal. Lateral and axial resolution is obtained from the focused

\section{Polymers with Intrinsic Microporosity Engineered for Hydrogen Storage}

Safe and efficient onboard hydrogen storage for cars cannot currently be based on reversible $\mathrm{H}_{2}$ adsorption in microporous materials like zeolites, carbon, or in materials based on a metalorganic framework, because the quantity of adsorbed $\mathrm{H}_{2}$ is insufficient. Organic polymers have heretofore not been considered, because their free volumes are generally much too small. Recently, however, N.B. McKeown of Cardiff University, P.M. Budd of the University of Manchester, D. Book of Birmingham University, and their colleagues have hypothesized that their recently reported "polymers of intrinsic microporosity" (PIMs) would be good candidates for $\mathrm{H}_{2}$ storage because they form solids with large, interconnected free volume with accessible internal surface areas in the range of $500-900 \mathrm{~m}^{2} / \mathrm{g}$. The rigidly contorted macromolecular structures formed by PIMs pack inefficiently and are a consequence of their fused-ring subunits, one of which typically contains a site of contortion, for example, a rigid, nonplanar unit.

As reported in a recent issue of Angewandte Chemie International Edition (Vol. 45, Issue 11, DOI: 10.1002/ anie.200504241; p. 1804), McKeown and co-researchers tailored the micropore structure of PIMs by using monomers that contain preformed cavities to provide appropriately sized sites for hydrogen adsorption. The researchers incorporated a bowl-shaped receptor monomer-cyclotricatechylene (CTC) — within a network PIM by using a benzodioxaneformation reaction between CTC and tetrafluoroterephthalonitrile, designating the new PIM as CTC-network-PIM. More conventional PIMs (PIM-1 and HATN-network-PIM; see Scheme I) were synthesized in order to compare gas adsorption properties. Structures for all three PIMs were confirmed by elemental analysis, infrared spectroscopy, and, in the case of the soluble PIM-1, ${ }^{1} \mathrm{H}$ and ${ }^{13} \mathrm{C}$ nuclear magnetic resonance. Nitrogen adsorption measurements showed that while all three PIMs have Brunauer-Emmett-Teller (BET) surface areas of about $800 \mathrm{~m}^{2} / \mathrm{g}$ with pore distributions biased in the range of $0.6-0.7 \mathrm{~nm}$, the bias for CTCnetwork-PIM is particularly dramatic, which the researchers attribute to the internal dimensions of the CTC subunit. This finding suggests to the researchers that the choice of monomer precursor can be used to tune the pore size distributions within PIMs.

Hydrogen adsorption is both rapid and reversible, which

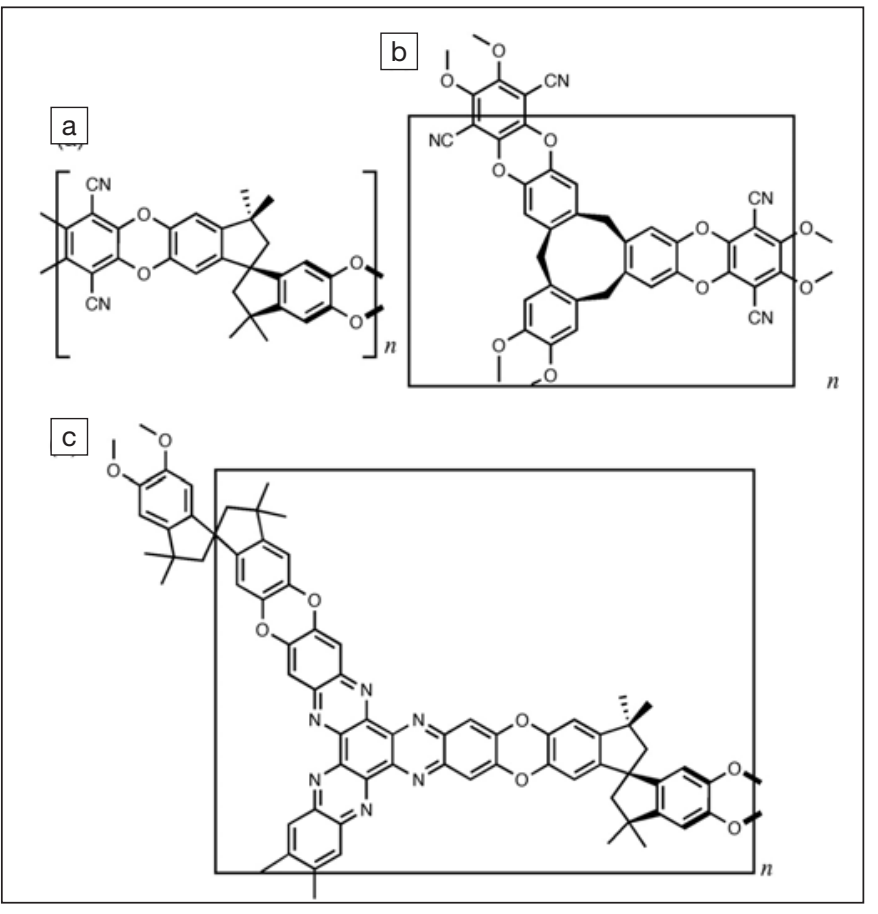

Scheme I. Soluble "polymers of intrinsic microporosity": (a) PIM-1, (b) HATN-network-PIM, and (c) CTC-network-PIM. Reproduced with permission from Angewandte Chemie International Edition 45 (11) (2006) DOl: 10.1002/anie.200504241; p. 1804). (c) 2006 Wiley-VCH Verlag GmbH \& Co KG.

the researchers expected for physisorption on a microporous material. All three PIMs adsorb a maximum of $1.4-1.7 \%$ by mass at pressures of $>10$ bar. The number of hydrogen molecules adsorbed per ring- 0.5 for PIM- $1,0.43$ for HATN-network-PIM, and 0.56 for CTC-network-PIM-suggests to the researchers that $\mathrm{H}_{2}$ adsorption is enhanced by the greater predominance of ultramicropores that result from the incorporation of the bowl-shaped CTC subunits. The researchers said that they "believe that optimization of PIMs for $\mathrm{H}_{2}$ adsorption presents additional opportunities towards achieving the U.S. Department of Energy's ambitious 2010 target of a practical storage system that holds $6.0 \% \mathrm{H}_{2}$ by mass."

STEVEN TROHALAKI 